\title{
Quality of life and self-rated health in relation to changes in fruit and vegetable intake and in plasma vitamins $C$ and $E$ in a randomised trial of behavioural and nutritional education counselling
}

\author{
Andrew Steptoe ${ }^{1 *}$, Linda Perkins-Porras ${ }^{1}$, Sean Hilton ${ }^{2}$, Elizabeth Rink ${ }^{2}$ and Francesco P. Cappuccio ${ }^{2}$ \\ ${ }^{1}$ Department of Epidemiology and Public Health, University College London, 1-19 Torrington Place, London WC1E 6BT, \\ $U K$ \\ ${ }^{2}$ Department of General Practice and Primary Care, St George's Hospital Medical School, Cranmer Terrace, London SW17 \\ ORE, $U K$
}

(Received 31 December 2003 - Revised 12 March 2004 - Accepted 17 March 2004)

\begin{abstract}
We have carried out a randomised trial comparing brief behavioural counselling with nutritional education counselling to increase fruit and vegetable consumption and associated biomarkers in adults from a low-income neighbourhood. The objective of the present analysis was to assess the impact of interventions on quality of life and health status, and associations between changes in fruit and vegetable consumption, plasma vitamins $\mathrm{C}$ and $\mathrm{E}$, and quality of life. Behavioural counselling and nutritional education counselling were carried out in 271 adults in two 15 min sessions in a primary-care setting. Physical and mental health status (medical outcome study short form 36) and selfrated health were assessed at baseline, 8 weeks and 12 months, and analysed on an intention-to-treat basis. Both groups reported increased fruit and vegetable consumption; plasma vitamin $\mathrm{E}$ and $\beta$-carotene also increased, with significantly greater changes in consumption and plasma $\beta$-carotene in the behavioural counselling condition. Physical and mental health status, and the proportion of participants in good self-rated health, increased in both groups to a similar extent. Individual differences in improvements in physical health status and selfrated health were correlated with increases in fruit and vegetable intake and in plasma vitamins $\mathrm{C}$ and $\mathrm{E}$, independently of age, gender, ethnicity, financial status, smoking, BMI and use of vitamin supplements. We conclude that participation in the present study was associated with improved health-related quality of life. Increases in fruit and vegetable intake and plasma vitamin levels may stimulate beneficial changes in physical health status in socio-economically deprived adults.
\end{abstract}

Dietary counselling: Quality of life: Plasma vitamins: Fruits and vegetables: Socio-economic status

Increasing fruit and vegetable consumption is a major objective of health-promotion programmes worldwide (World Health Organization, 2002; Department of Health, 2003). Consumption is greater in higher socio-economic status groups, so increasing intake in lower income sectors may help reduce socio-economic disparities in health (Henderson et al. 2002). We have recently conducted a randomised controlled trial comparing brief behavioural counselling and nutritional education counselling in a primary-care setting in a low-income, ethnically mixed neighbourhood. Increases in fruit and vegetable consumption over 12 months were reported by both groups, and were corroborated by changes in plasma $\beta$-carotene and vitamin E (Steptoe et al. 2003a). The increases in intake and in plasma $\beta$-carotene were significantly greater following behavioural counselling after adjusting for gender, age, income, ethnicity, smoking status and baseline stage of change.

An important issue in evaluating interventions is whether they have adverse effects on quality of life, despite being efficacious for the target health problem. Quality of life evaluations are conventional in pharmaceutical trials, but have been included less often in dietary studies. This situation has changed in recent years, and several interventions with dietary components have incorporated quality of life measures into their assessment batteries (Plaisted et al. 1999; Corle et al. 2001; Pierce et al. 2002; Toobert et al. 2002; Miller et al. 2003). It cannot be assumed that dietary interventions are free of adverse effects on quality of life and health status. There were fears 10 years ago that cholesterol-lowering through fat reduction might induce depressive mood (Muldoon et al. 1990), although subsequent trials allayed these concerns (Muldoon et al. 2001). Changes in digestive symptoms that could adversely affect quality of life have been reported with increased fruit and vegetable intake (Smith-Warner et al. 2000). In addition, it is possible that attempts to change eating habits in social settings may lead to embarrassment and negative social interactions (Steptoe et al. 2003b). The first aim of the present analysis was, therefore, to evaluate the effects of participation in behavioural or nutritional

Abbreviation: SF-36, medical outcome study short form 36

* Corresponding author: Professor Andrew Steptoe, fax +44 207916 8542, email a.steptoe@ucl.ac.uk 
education counselling for increasing fruit and vegetable intake on health-related quality of life and self-rated health.

We were also interested in whether changes in fruit and vegetable intake and in biomarkers such as plasma vitamin $\mathrm{C}$, vitamin $\mathrm{E}$ and $\beta$-carotene were associated with changes in health status. It has been claimed that vitamin supplementation positively affects subjective well-being (Benton et al. 1995), although this has not been replicated consistently (Barringer et al. 2003). Inverse associations between plasma or serum vitamin $\mathrm{E}$ and depressive symptoms have been reported in some studies, but not all (Shibata et al. 1999; Maes et al. 2000; Tiemeier et al. 2002). Vitamins C and $\mathrm{E}$ are thought to have favourable effects on neural function and cognitive performance, but the evidence is again inconsistent (Paleologos et al. 1998; Martin et al. 2002; Morris et al. 2002). The second aim was to test whether changes in reported fruit and vegetable intake and in biomarkers over 8 weeks and 12 months were associated with health status assessed using the medical outcome study short form 36 (SF-36) and measures of self-rated health.

\section{Methods}

\section{Participants}

Men and women ( $n$ 271) aged 18-70 years were randomly recruited from the patient registers of a primary-health centre in South London (Steptoe et al. 2003a). The health centre is in a deprived inner-city area. Individuals with serious illnesses and women who were pregnant or planned to become pregnant within the next 12 months were excluded. A total of 459 patients expressed interest in the study, but 188 were excluded on these criteria or because they were found on later enquiry to have high incomes.

\section{Study design}

This parallel-group randomised trial compared brief behavioural dietary counselling with nutritional education counselling matched for professional contact time (Steptoe et al. 2003a). Recruitment, assessments and interventions were carried out by research nurses. The interventions were delivered in two $15 \mathrm{~min}$ individual counselling sessions carried out at a 2-week interval, supported by written information. Assessments, including reported fruit and vegetable intake, plasma measures of $\beta$-carotene, vitamin $\mathrm{E}$ ( $\alpha$-tocopherol) and vitamin $\mathrm{C}$ (ascorbic acid), were performed at baseline before randomisation, and after 8 weeks and 12 months. We assessed quality of life at baseline, 8 weeks and 12 months. The study was approved by the Wandsworth Local Research Ethics Committee.

\section{Counselling methods}

The nutrition education counselling group received education about the importance of increasing consumption, emphasising the beneficial effects of nutritional constituents and the way these act biologically to maintain health. The bioactive constituents of fruits and vegetables were described in lay terms, together with the range of effects that they have on body processes. The recommendation from the UK Department of Health (2003) to eat five portions of a variety of fruits and vegetables per $\mathrm{d}$ was emphasised. The behavioural counselling included abbreviated nutritional education, but in addition involved counselling founded on social learning theory and the stage of change model, so methods of encouraging behaviour change varied with the motivational readiness of the individual (Hunt \& Hillsdon, 1996; Prochaska \& Velicer, 1997). Readiness to change, dietary knowledge and perceived barriers were assessed, so that counselling could be tailored to the individual. Thus, the counselling for an individual who was not convinced of the benefits of fruit and vegetable consumption emphasised attitude change, while counselling for those ready to change focused on specific behavioural goals and the practical implementation of change. Social reinforcement was used to foster self-efficacy for change and the attainment of short-term and long-term goals. Written material addressed common barriers to change, and suggested ways of overcoming these. Both of the research nurses conducted nutritional and behavioural counselling after training from experienced behavioural scientists, and sessions were audio-taped to monitor the quality of interventions.

\section{Measures}

We measured fruit and vegetable consumption with a twoitem frequency questionnaire adapted from previous research (Wardle et al. 2000). Participants were asked how many servings of fruits and how many portions of vegetables they ate on a typical day, and were given detailed information about portion sizes. Potatoes were excluded, as is standard practice in UK research, and one serving of fruit juice was included. This measure was validated against plasma and urinary biomarkers of fruit and vegetable intake at baseline, with evidence that self-reported fruit intake was correlated with plasma levels of vitamin $C$ and $\beta$-carotene and with urinary $\mathrm{K}$ excretion, while fruit and vegetable intake was associated with plasma vitamin $\mathrm{C}$ and $\mathrm{K}$ excretion expressed as total daily excretion and as the $\mathrm{K}$ :creatinine ratio (Cappuccio et al. 2003).

Quality of life was assessed using the SF-36 health status measure, adapted slightly for use in the UK (Ware \& Sherbourne, 1992; Jenkinson et al. 1996). The SF-36 assesses eight domains of health-related quality of life: physical function; role limitations due to physical problems; role limitations due to emotional problems; social functioning; general mental health; vitality; pain; general health perceptions. Each domain is scaled so that 0 represents the lowest and 100 the highest possible level of function. Scores for the eight domains at baseline were calculated. We evaluated change in quality of life by following the procedure advocated by Ware et al. (1994) of calculating physical health and mental health status summary measures. Physical health status is calculated by averaging scores on physical function, role limitations due to physical problems, pain and general health perception measures, while mental health status is calculated by averaging scores on the role limitations due to emotional problems, social functioning, general mental health and vitality measures.

Self-rated health was assessed with a single item: 'In general, would you say your health is excellent/very 
good/good/fair/poor'. Simple measures of this type have been shown to predict health status and mortality in a large number of studies (Idler \& Benyamini, 1997) and are suitable across the socio-economic gradient (Burstrom \& Fredlund, 2001).

Non-fasting venous samples were analysed to determine plasma vitamin C (ascorbic acid), vitamin E ( $\alpha$-tocopherol) and $\beta$-carotene. Blood for vitamin $C$ analysis was collected in citrate, protected from light, cold spun and plasma added to metaphosphoric acid $(100 \mathrm{ml} / \mathrm{l})$. Levels were estimated using a fluorometric assay (Vuilleumier \& Keck, 1989). Vitamin $\mathrm{E}$ and $\beta$-carotene were measured concurrently by normal-phase HPLC (Armstrong et al. 1997).

\section{Procedure}

The patients who agreed to take part in the study completed baseline quality of life measures before attending an individual assessment session in the health centre. Information concerning ethnicity, marital status, educational attainment, income, smoking status, stage of readiness for change and vitamin supplements was obtained by questionnaire. At the assessment session, fruit and vegetable intake was recorded, blood samples were drawn, and anthropometric measures and a medical history were obtained. Randomisation took place after the baseline assessment using a minimisation procedure to ensure a balance across groups in age, gender, ethnic background and smoking (Treasure \& MacRae, 1998). The first $15 \mathrm{~min}$ counselling session followed immediately, and the second counselling session 2 weeks later. A third $15 \mathrm{~min}$ encouragement and support session was scheduled after 6 months. Measures of fruit and vegetable intake, biomarkers and quality of life were repeated 8 weeks after the baseline and then after 12 months.

\section{Statistical analysis}

The 8-week assessment was completed by $245(90.4 \%)$ of participants, and the 12-month assessment by 218 (80.4\%). The number of people who failed to complete follow-up assessments did not differ between groups. All analyses were carried out on an intention-to-treat basis, including data from all participants entered into the trial, irrespective of whether they completed all assessments or dropped out of the study. We assumed no change in individuals who did not complete follow-up assessments, so carried forward the baseline measure for missing data at 8 weeks and 12 months. Data on fruit and vegetable intakes were available from all 271 participants, $\beta$-carotene from 268 (99\%), vitamin E from 266 (98\%) and vitamin C from $265(98 \%)$.

The attributes of the behavioural counselling and nutritional education counselling groups at baseline were compared by ANOVA and $\chi^{2}$ tests as appropriate. Financial status was categorised according to whether income was greater than or less than $£ 400$ per week. This is less than the national average gross income of $£ 480$ per week in 1999-2000, and close to the average for lower socio-economic status employment categories (Down, 2000). Of the participants, 177 of $271(65 \%)$ fell into the lower income category. SF-36 ratings for all eight health status domains. as well as summary physical and mental health scales. were compared between groups and also with the values obtained in the Oxford Healthy Life Survey (UK population survey of 13042 randomly selected men and women aged 18-64 years; Jenkinson et al. 1993). Selfrated health was divided into fair or poor health, and good health or better.

We analysed changes in quality of life over the trial using repeated measures ANOVA of physical health and mental health status summary scores, and the proportion of people who rated their health as good or better. These analyses included group (behavioural, nutritional education counselling) and gender as between-subject factors, and time (baseline, 8 weeks, 12 months) as the within-subject factor. The Greenhouse-Geisser correction was applied when appropriate and post hoc comparisons were carried out using Fisher's least significant difference procedure.

Associations between predictors (fruit and vegetable consumption and biomarkers) and physical and mental health status summary measures were analysed with a series of multiple regressions. These regression analyses included covariates that we have previously identified as related to changes in reported consumption and biomarkers (Steptoe et al. 2003a). In the analyses involving fruit and vegetable consumption, the covariates were age, gender, ethnicity (coded as a binary variable), financial status (coded as higher or lower income), smoking status (binary variable) and BMI (continuous variable). In the analyses involving biomarkers, the covariates included these factors plus use of vitamin supplements. Unstandardised linear regression coefficients for the predictor variables of interest (fruit and vegetable consumption, vitamin $C$, vitamin $E$ or $\beta$-carotene) adjusted for covariates are presented with $95 \% \mathrm{CI}$. We analysed changes in self-rated health by calculating whether there had been a shift from fair or poor health to good or better health between baseline and 8 weeks or 12 months. These data were analysed using logistic regression, and odds ratios with $95 \% \mathrm{CI}$ are presented.

\section{Results}

The attributes of participants in the two groups at baseline are summarised in Table 1. Overall, $61.3 \%$ of participants were women, with no gender difference between groups. About one-third came from ethnic minorities, predominantly black African and black Afro-Caribbean. Two-thirds were below the low-income criterion, with one-third being in receipt of welfare benefits. There were no differences between groups in any of these characteristics or in BMI, smoking habits or the proportion using vitamin supplements. The treatment groups did not differ in fruit and vegetable consumption or in biomarkers at baseline. The SF-36 indicated only moderate functioning in all domains, and $22.5 \%$ were in poor or fair health. Average SF-36 ratings were significantly below those of the previously published population survey in all eight domains $(P<0 \cdot 001)$.

\section{Responses to the interventions}

The effects of behavioural and nutritional education counselling have been detailed elsewhere, so only summary results will be presented (Steptoe et al. 2003a,b). The number 
Table 1. Attributes of the behavioural and nutritional counselling groups at baseline (Mean values and standard deviations)

\begin{tabular}{|c|c|c|c|c|}
\hline & \multicolumn{2}{|c|}{$\begin{array}{l}\text { Behavioural counselling } \\
\qquad(n 136)\end{array}$} & \multicolumn{2}{|c|}{$\begin{array}{l}\text { Nutritional education } \\
\text { counselling }(n 135)\end{array}$} \\
\hline & Mean & SD & Mean & $S D$ \\
\hline Men/women $(n)$ & $54 / 82$ & & $51 / 84$ & \\
\hline Age & $43 \cdot 3$ & $13 \cdot 8$ & 43.2 & $14 \cdot 0$ \\
\hline \multicolumn{5}{|l|}{ Ethnicity: } \\
\hline White $^{*}$ & 94 & $70 \cdot 1$ & 96 & $72 \cdot 2$ \\
\hline Black* $^{*}$ & 37 & $27 \cdot 6$ & 32 & $24 \cdot 1$ \\
\hline Asian* & 3 & $2 \cdot 2$ & 5 & $3 \cdot 8$ \\
\hline Household income $<£ 400$ per week* & 91 & 69.5 & 86 & $66 \cdot 2$ \\
\hline In receipt of welfare benefits* & 50 & 37.3 & 43 & $32 \cdot 8$ \\
\hline BMI & 25.5 & 4.9 & $26 \cdot 3$ & $5 \cdot 8$ \\
\hline Current smokers* & 47 & $34 \cdot 6$ & 44 & $32 \cdot 6$ \\
\hline Taking vitamin supplements* & 40 & $32 \cdot 3$ & 38 & 30.4 \\
\hline Portions of fruit and vegetables ( $n$ per d) & 3.67 & $2 \cdot 00$ & 3.60 & 1.81 \\
\hline Plasma vitamin $C(\mu \mathrm{mol} / /)$ & 78.0 & $33 \cdot 0$ & $75 \cdot 6$ & $33 \cdot 3$ \\
\hline Plasma vitamin E ( $\mu \mathrm{mol} / /)$ & 27.4 & 10.9 & $25 \cdot 6$ & $11 \cdot 3$ \\
\hline Plasma $\beta$-carotene $(\mu \mathrm{mol} / \mathrm{l})$ & 0.92 & 0.68 & 0.90 & 0.62 \\
\hline \multicolumn{5}{|l|}{ SF-36 } \\
\hline Physical function & $82 \cdot 2$ & $22 \cdot 7$ & 81.6 & $24 \cdot 1$ \\
\hline Physical role limitations & $76 \cdot 0$ & 37.6 & 73.7 & $36 \cdot 8$ \\
\hline Emotional role limitations & 71.8 & 38.7 & $70 \cdot 0$ & 37.2 \\
\hline Social functioning & $80 \cdot 8$ & 21.9 & 79.4 & 23.4 \\
\hline General mental health & 68.7 & $18 \cdot 0$ & $67 \cdot 1$ & $17 \cdot 6$ \\
\hline Vitality & $55 \cdot 8$ & 21.0 & 53.1 & $20 \cdot 8$ \\
\hline General health perceptions & 62.6 & $22 \cdot 4$ & 65.0 & 21.6 \\
\hline Pain & 71.5 & $25 \cdot 2$ & 73.0 & $23 \cdot 7$ \\
\hline Physical health & $73 \cdot 1$ & $22 \cdot 1$ & 73.3 & 20.9 \\
\hline Mental health & $69 \cdot 2$ & $21 \cdot 1$ & 67.4 & $20 \cdot 3$ \\
\hline Self-rated health good or better* & 104 & 76.4 & 105 & 77.7 \\
\hline
\end{tabular}

SF-36, Medical outcome short study form 36 (Ware \& Sherbourne, 1992; Jenkinson et al. 1996).

${ }^{*} n$ and $\%$.

of portions of fruits and vegetables consumed per d increased over 12 months by 1.49 (95\% CI $1.12,1.86)$ in the behavioural counselling group and by 0.87 (95\% CI $0.50,1 \cdot 25)$ in the nutritional counselling group, a mean difference of 0.62 (95\% CI $0.09,1.13 ; P<0.05$ ) adjusted for gender, age, ethnicity, income, smoking and baseline stage of change. The proportion of participants consuming five or more portions per d increased by 42.2 (95\% CI 33.1, 51.2) $\%$ in the behavioural counselling group and 26.8 (95\% CI $17 \cdot 6,36 \cdot 0) \%$ in the nutritional counselling group (difference $15.4(95 \%$ CI $2.5,28.3) \%, P<0.05)$. The plasma concentration of vitamin $\mathrm{E}$ increased over 12 months in both groups (mean values 8.81 (95\% CI 7.1, 10.5) and 7.30 (95\% CI 5.58, 9.02) $\mu \mathrm{mol} / \mathrm{l}$ for behavioural and nutritional counselling groups respectively), with no difference between the groups. There were also increases in $\beta$-carotene in both groups (mean values 1.20 (95\% CI 1.08, 1.33) and $1.04(95 \%$ CI $0.94,1.15) \mu \mathrm{mol} / 1$ respectively), with a larger increase in the behavioural counselling group (mean difference 0.16 (95\% CI 0.001, 0.340) $\mu \mathrm{mol} / 1, P<0.05$ ). Plasma vitamin $\mathrm{C}$ did not change in either group over the 12-month study period.

\section{Changes in quality of life over the study}

There was a main effect of time $(P<0 \cdot 001)$, but no group or gender differences in the analysis of the physical health status summary measure (Fig. 1). Physical health status increased between baseline and 8 -week assessments $(P<0.001)$, and remained elevated at 12 months in both groups $(P<0.001)$. There was no change between 8 weeks and 12 months. A similar pattern was observed for the mental health status summary measure, with a significant change over time $(P<0.001)$ due to increases between baseline and 8 weeks (Fig. 1). The behavioural and nutritional education counselling groups did not differ in the magnitude of increases.

The proportion of participants who reported that their health was good, very good or excellent increased over time from an average of $77.1 \%$ at baseline to $83.1 \%$ after 8 weeks and $82.3 \%$ after 12 months $(P<0 \cdot 01)$. Post hoc tests indicated that the proportion in good or better health differed between baseline and 8 weeks $(P<0.05)$ and between baseline and 12 months $(P<0.05)$, but not between 8 weeks and 12 months (Fig. 2). This pattern did not differ between groups, and there were no gender differences at any point.

\section{Fruit and vegetable intake and quality of life}

Baseline levels of fruit and vegetable consumption were positively associated with physical health status and mental health status summary scores, but not with selfrated health. The regressions of fruit and vegetable intake $v$. physical health status (B 1.45 (95\% CI 0.13, 2.77), $P<0.05$ ) and mental health status (B 1.47 (95\% CI 0.16, $2.78), P<0.05)$ were independent of age, gender, ethnicity, income, smoking and BMI. Self-rated health at baseline was not associated with fruit and vegetable consumption. 
(A)

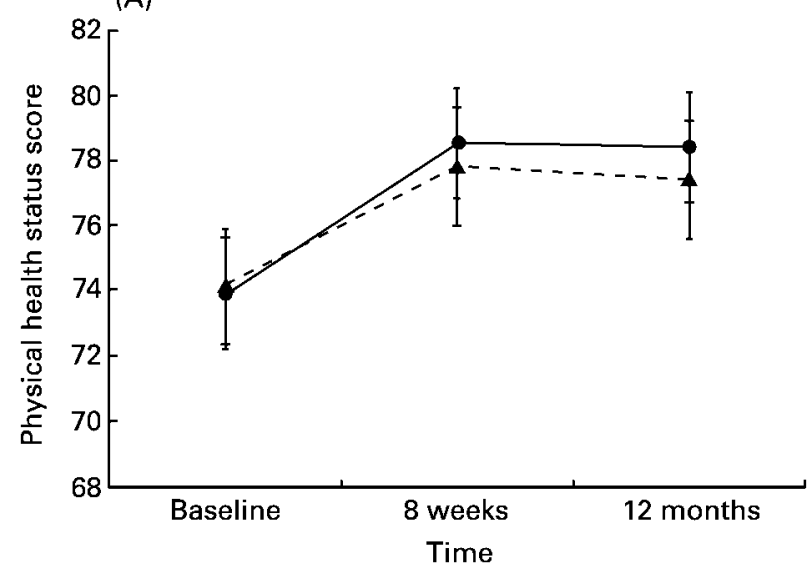

(B)

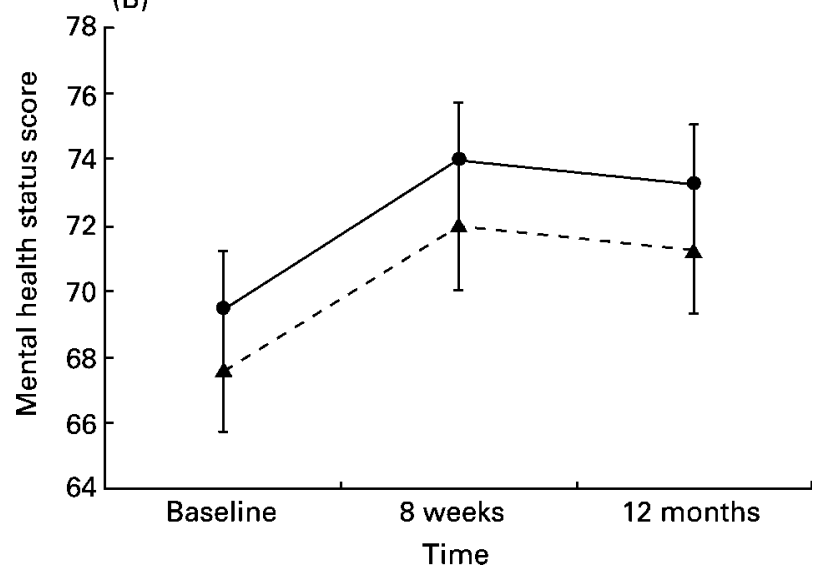

Fig. 1. Mean ratings on the physical $(A)$ and mental $(B)$ health status summary scales at baseline, 8 weeks and 12 months in the behavioural $(-)$ and nutritional education (--) counselling conditions. All values are adjusted for gender, age, ethnicity, income, smoking and BMI. For details of subjects and procedures, see Table 1 and p. 178. Values are means with their standard errors shown by vertical bars.

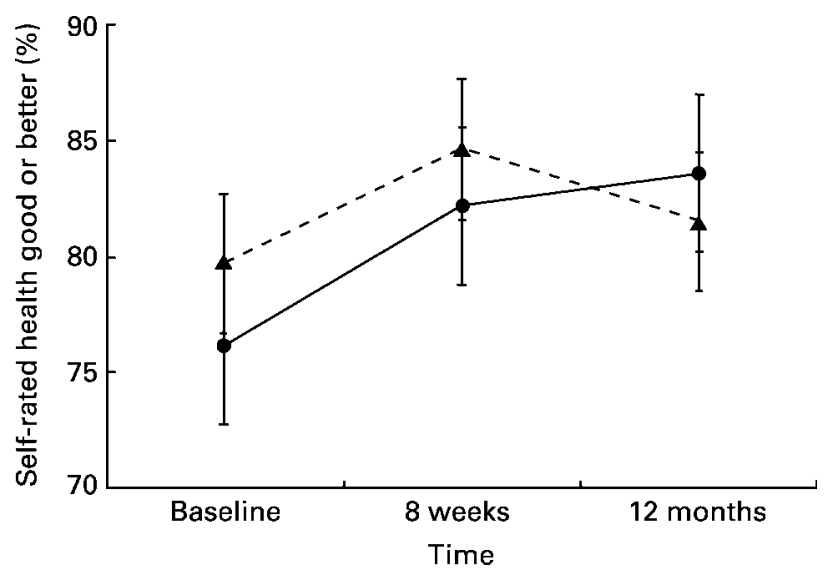

Fig. 2. Proportion of participants rating their health as good, very good or excellent at baseline, 8 weeks and 12 months in the behavioural (-) and nutritional education (--) counselling groups. All values are adjusted for gender, age, ethnicity, income, smoking and BMI. For details of subjects and procedures, see Table 1 and p. 178. Values are means with their standard errors shown by vertical bars.
The change in physical health status summary scores between baseline and 8 weeks was positively associated with change in fruit and vegetable consumption independently of covariates (B $0.80 \quad(95 \%$ CI $0.11,1.50)$, $P<0 \cdot 05)$. The effect remained significant after additional control for fruit and vegetable consumption at baseline, so was not due to individuals with high initial intake showing larger changes in consumption and improvements in physical health status. Change in fruit and vegetable consumption remained associated with changes in physical health status over 12 months (B 1.17 (95\% CI 0.37, $2 \cdot 02), P<0 \cdot 01)$. Thus, individuals who reported greater increases in fruit and vegetable consumption also showed greater improvements in physical health status over the study period.

There was no significant association between changes in mental health status summary scores and fruit and vegetable consumption. In the case of self-rated health, effects at 8 weeks were not significant. However, the likelihood of showing an improvement from fair or poor to good health between baseline and 12 months was related to increases in fruit and vegetable consumption. The odds ratio adjusted for age, gender, ethnicity, income, smoking and BMI of reporting an improvement in self-rated health was 1.35 (95\% CI $1.08,1.69 ; P<0.01)$ per unit increase in number of daily servings of fruits and vegetables. This effect remained significant after controlling for baseline fruit and vegetable intake.

\section{Plasma vitamins and quality of life}

The associations between baseline quality of life measures and baseline plasma vitamin $\mathrm{E}$ were not significant. But the change in vitamin $\mathrm{E}$ concentration between baseline and 12 months was positively related to changes in physical health status summary scores independently of age, gender, ethnicity, income, smoking, BMI and use of vitamin supplements at either time point (B 0.15 (95\% CI 0.01, $0 \cdot 30), P<0 \cdot 05)$. Similarly, the odds of an improvement in self-rated health were associated with increased vitamin E over 12 months (odds ratio 1.05 (95\% CI 1.01, 1.09), $P<0.01)$. Changes in vitamin $\mathrm{E}$ between baseline and 8 weeks were related to the improvement in self-rated health over this time period as well (odds ratio 1.04 (95\% CI 1.00, 1.08), $P<0.05$ ), but not with the change in physical health status. We did not observe any associations between vitamin $\mathrm{E}$ and mental health status.

We found that the likelihood of good or better self-rated health was positively associated with baseline vitamin $\mathrm{C}$, independently of covariates (odds ratio 1.02 (95\% CI $1.01,1.03), P<0.005)$. Plasma vitamin $\mathrm{C}$ concentration at baseline was not related to physical or mental health status ratings. However, there was a positive association between changes in plasma vitamin $\mathrm{C}$ over 8 weeks and improvements in physical health status summary ratings independently of covariates (B 0.97 (95\% CI 0.41, 0.15), $P<0.001)$. Thus, participants who showed larger increases in vitamin $\mathrm{C}$ concentration also reported greater improvements in physical health status. This association did not persist at 12 months, and no associations of vitamin $\mathrm{C}$ 
with changes in mental health status summary scores or self-rated health were identified.

Analyses were also carried out relating quality of life measures with $\beta$-carotene, but no significant associations were present.

\section{Discussion}

The present study was carried out in a primary-care centre in a low-income neighbourhood. One-third of participants were on very low incomes and in receipt of welfare benefits, while another one-third did not receive benefits, but were below our threshold for low income. Analyses not described here indicate that the interventions were equally effective when higher income participants were excluded (Steptoe et al. 2003a). Ethnic minority adults are often under-represented in research studies in the UK, but the $28.4 \%$ of participants being black or Asian compares favourably with the estimated $22 \%$ of non-white residents of the London borough in which the present study was carried out, according to the 2001 National Census. The fruit and vegetable intake of 3.64 portions perd at baseline is comparable with the average of 3.58 reported in the UK 1999 National Food Survey (Ministry of Agriculture, Fisheries \& Food, 2000), although slightly greater than estimates in the 20002001 National Diet and Nutrition Survey (Henderson et al. 2002). Baseline levels of plasma vitamin $C$, vitamin $\mathrm{E}$ and $\beta$-carotene were comparable with those reported in other UK studies (Armstrong et al. 1997). The proportion of individuals taking vitamin supplements was similar to that described in the Health Survey for England (Erens \& Primatesta, 1999).

Physical health status, mental health status and self-rated health all improved over the course of the study (Figs 1 and 2 ). These analyses were carried out on an intention to treat basis, so favourable responses were not due to the selective effects of only including data from subjects who successfully completed the trial. The changes took place during the intensive phase of the interventions (between baseline and 8 weeks), with little further change over the subsequent 10 months. We limited analyses to physical health and mental health summary scales rather than analysing all eight domains of the SF-36 separately, in order to reduce the number of analyses and risks of type I error. The quality of life responses cannot be attributed to reductions in body weight, since no significant changes were observed.

Few beneficial effects on quality of life have been reported in other dietary intervention studies. For example, the SF-36 was completed before and after 8 weeks of intervention in eighty-three participants in the Dietary Approaches to Stop Hypertension (DASH) trial (Plaisted et al. 1999). Few changes were observed in any of the quality of life domains, except that vitality increased in all three conditions (control, fruit and vegetable, and combination diets), body pain improved in the combination diet condition, and ratings of change in health improved in the two intervention conditions, but not the control conditions. Corle et al. (2001) included an amalgam of items from the SF-36 and the general well-being scale to assess quality of life in the Polyp Prevention Trial. No changes or differences between groups were observed, despite differences in fat and fibre intake and in fruit and vegetable consumption between the dietary intervention and control groups. In a study of women with type 2 diabetes, a comprehensive lifestyle management intervention involving a Mediterranean diet, stress management, physical activity, group support and smoking cessation produced greater improvements in glucose metabolism, BMI and plasma fatty acid concentrations than in control subjects over 6 months (Toobert et al. 2003). There were, however, no differences and no changes in physical or mental health assessed with the SF-12.

One explanation for the greater effects in the present study is that participants began with relatively poor health status (Table 1). Scores in all eight domains of the SF-36 were lower than in population surveys in the UK. This is likely to be due to the low socio-economic status of participants, since it is known that self-rated health and health-related quality of life are poorer in lower status groups (Jenkinson et al. 1993). By contrast, three scales of the SF-36 (physical, emotional and social role functioning) were near maximum in all participants in the DASH sub-study, so little further improvement was possible (Plaisted et al. 1999).

Both counselling conditions stimulated increases in reported fruit and vegetable consumption over the 12month follow-up period, accompanied by increases in plasma vitamin $\mathrm{E}$ and $\beta$-carotene. The changes in fruit and vegetable intake and $\beta$-carotene were greater with behavioural than nutritional education counselling, from which we conclude that the provision of personalised advice based on behavioural principles supplemented the effects of nutritional education alone. The counselling was carried out by research nurses rather than by dietitians for two reasons. First, the research nurses also conducted physical assessments, including blood sampling and urinary measurement. Second, we anticipated that these methods might be most readily applied in primary care by practice nurses. It is interesting that despite the greater response from the behavioural counselling group in terms of reported consumption and plasma $\beta$-carotene, there was no difference between conditions in the changes in physical health status and quality of life. This indicates that the improvement in quality of life was not linked with the specific procedures used in one condition or another, but may have been a response to changes in diet.

This argument is supported by the second notable set of findings, concerning the relationships between fruit and vegetable intake and quality of life measures. The observation that consumption at baseline was associated with physical and mental health status is not unexpected, given the presumed health benefits of a diet rich in fruits and vegetables. More interesting is the observation that changes in fruit and vegetable consumption were associated with improvements in physical health status and self-rated health. The physical health status effects were significant at 8 weeks and 12 months, while the self-rated health effect emerged only at 12 months. These results indicate that individuals who reported greater increases in fruit and vegetable consumption also showed greater 
improvements in physical health and well-being. A Danish population study has previously demonstrated that poor self-rated health is more common among adults who do not consume good diets characterised by high fruit and vegetable intakes (Osler et al. 2001). Furthermore, over a 15-year follow-up period, mortality was associated both with poor self-rated health and poorer diets in the present study.

Scores on the mental health status measure improved in both groups during the trial, but were not associated with changes in fruit and vegetable consumption. This suggests that the mental health changes were non-specific responses to participation, rather than specific effects of dietary modification. It is possible that participants felt better for deciding to take part in the study, whether or not the outcome was favourable. Participation involved periodic support from research nurses together with encouragement of any appropriate behaviour changes, and these may have been sufficient to stimulate improvements in mental well-being.

Support for more specific effects of dietary change on physical health status and self-rated health was provided by analyses of plasma biomarkers. Increases in vitamin $\mathrm{E}$ at both 8 weeks and 12 months were associated with improved self-rated health, while changes in vitamin $E$ at 12 months correlated with improved physical health status. Plasma vitamin $\mathrm{C}$ was associated with self-rated health at baseline. There was no significant change in plasma vitamin $\mathrm{C}$ on average, since some individuals showed increases while others showed decreases over the study period. The changes between baseline and 8 weeks correlated with the magnitude of improvements in physical health status. All these effects were independent of gender, age, ethnicity, income, smoking, BMI and the use of vitamin supplements, so may be direct consequences of changes in diet stimulating plasma vitamin responses.

Few controlled studies have related changes in vitamin intake with quality of life. The main interest has been in the impact of vitamins on cognitive function and mood (Martin et al. 2002). For example, Morris et al. (2002) found that cognitive decline over 3 years in a cohort of older adults was reduced in those with higher vitamin $\mathrm{E}$ intake. An inverse association between vitamin $\mathrm{E}$ and depression has been described in some studies (Shibata et al. 1999; Maes et al. 2000), but not in others once potential confounders had been taken into account (Tiemeier et al. 2002). Cross-sectional associations between vitamin $\mathrm{C}$ concentration and cognitive function have also been observed (Ortega et al. 1997), but longitudinal results have been inconsistent (Paleologos et al. 1998; Morris et al. 2002). A recent 12-month randomised controlled trial of vitamin and mineral supplements $v$. placebo in community-dwelling adults showed no significant changes or differences between groups in physical or mental health status assessed with the SF-12 (Barringer et al. 2003).

Our present results suggest that long-term changes in fruit and vegetable intake and plasma vitamin E concentration may have beneficial effects on physical health status and self-rated health. A review of the literature on self-rated health published until 1996 found that poor self-rated health predicted lower survival in twenty-three of twenty-seven studies even when known risk factors had been taken into account (Idler \& Benyamini, 1997). This pattern has been replicated in more recent investigations (Kawada, 2003), and self-rated health also predicts future functional limitations (Idler et al. 2000). Physical functioning ratings predict adverse health outcomes as well. For example, Franks et al. (2003) analysed 5-year mortality data from 19812 participants in the National Medical Expenditure Survey in the USA. Lower SF-20 scores on physical functioning, health perception and role functioning scales predicted reduced survival, with particularly strong effects among younger individuals. The changes in self-rated health and physical health status reported in this study may therefore be consequential in terms of broader health outcomes.

The present study was limited to residents in an ethnically mixed, low-income urban area, and may not be applicable to rural populations in other parts of the world. We have discussed issues of selection elsewhere (Steptoe et al. 2003a), but since results were only obtained from adults who agreed to participate in the trial, patterns might be different in the population at large. Only a summary measure of fruit and vegetable consumption was obtained, so detailed nutritional analyses could not be conducted and estimates of intake were not as precise as with more detailed instruments. Nonetheless, the results indicate that encouraging increases in fruit and vegetable intake in low-income populations does not have adverse effects on physical or mental health status, and may indeed lead to positive general health gains. The associations between individual differences in changes in consumption, plasma vitamin concentrations and quality of life suggest that modifying nutritional intake may have favourable effects on physical health status. Utilisation of these methods may help reduce socio-economic disparities in health behaviour and health.

\section{Acknowledgements}

This research was supported by grant 121695 in the Department of Health/Medical Research Council Nutrition Programme, UK. The views expressed are those of the authors, and not the UK Department of Health. We are grateful for the help of Catherine McKay and Martin Lipscombe in data collection, and Rosie Savage and staff and patients at the Falcon Road Health Centre for their cooperation.

\section{References}

Armstrong NC, Paganga G, Brunner E, et al. (1997) Reference values for alpha-tocopherol and beta-carotene in the Whitehall II study. Free Radic Res 27, 207-219.

Barringer TA, Kirk JK, Santaniello AC, Foley KL \& Michielutte R (2003) Effect of a multivitamin and mineral supplement on infection and quality of life. A randomized, double-blind, placebo-controlled trial. Ann Intern Med 138, $365-371$.

Benton D, Haller J \& Fordy J (1995) Vitamin supplementation for 1 year improves mood. Neuropsychobiology 32, 98-105.

Burstrom B \& Fredlund P (2001) Self rated health: Is it as good a predictor of subsequent mortality among adults in lower as well 
as in higher social classes? J Epidemiol Community Health 55, $836-840$

Cappuccio FP, Rink E, Perkins-Porras L, McKay C, Hilton S \& Steptoe A (2003) Estimation of fruit and vegetable intake using a two-item dietary questionnaire: a potential tool for primary health care workers. Nutr Metab Cardiovasc Dis 13, 12-19.

Corle DK, Sharbaugh C, Mateski DJ, et al. (2001) Self-rated quality of life measures: effect of change to a low-fat, high-fiber, fruit and vegetable enriched diet. Ann Behav Med 23, 198-207.

Department of Health, (2003) Five a Day: Increasing Fruit and Vegetable Consumption - A National Priority. http://www. doh.gov.uk/fiveaday

Down D (2000) Family Spending: A Report on the 1999-2000 Family Expenditure Survey. London: The Stationery Office.

Erens B \& Primatesta P (1999) Health Survey for England: Cardiovascular Disease '98. London: The Stationery Office.

Franks P, Gold MR \& Fiscella K (2003) Sociodemographics, selfrated health, and mortality in the US. Soc Sci Med 56, 2505-2514.

Henderson L, Gregory J \& Swan G (2002) The National Diet and Nutrition Survey: Adults Aged 19 to 64 years. Volume 1: Types and Quantities of Foods Consumed. London: The Stationery Office.

Hunt P \& Hillsdon M (1996) Eating and Exercise Behaviour: A Handbook for Professionals. Oxford: Blackwell Science.

Idler EL \& Benyamini Y (1997) Self-rated health and mortality: a review of twenty-seven community studies. J Health Soc Behav 38, 21-37.

Idler EL, Russell LB \& Davis D (2000) Survival, functional limitations, and self-rated health in the NHANES I Epidemiologic Follow-up Study, 1992. Am J Epidemiol 152, 874-883.

Jenkinson C, Coulter A \& Wright L (1993) Short form 36 (SF36) health survey questionnaire: normative data for adults of working age. Br Med J 306, 1437-1440.

Jenkinson C, Layte R, Wright L \& Coulter A (1996) The U.K. SF-36: An Analysis and Interpretation Manual. Oxford: Health Services Research Unit.

Kawada T (2003) Self-rated health and life prognosis. Arch Med Res 34, 343-347.

Maes M, De Vos N, Pioli R, et al. (2000) Lower serum vitamin E concentrations in major depression. Another marker of lowered antioxidant defenses in that illness. J Affect Disord 58, 241-246.

Martin A, Cherubini A, Andres-Lacueva C, Paniagua M \& Joseph J (2002) Effects of fruits and vegetables on levels of vitamins $\mathrm{E}$ and $\mathrm{C}$ in the brain and their association with cognitive performance. J Nutr Health Aging 6, 392-404.

Miller GD, Rejeski WJ, Williamson JD, et al. (2003) The Arthritis, Diet and Activity Promotion Trial (ADAPT): design, rationale, and baseline results. Control Clin Trials 24, 462-480.

Ministry of Agriculture, Fisheries and Food (2000) National Food Survey 1999. London: The Stationery Office.

Morris MC, Evans DA, Bienias JL, Tangney CC \& Wilson RS (2002) Vitamin E and cognitive decline in older persons. Arch Neurol 59, 1125-1132.

Muldoon MF, Manuck SB \& Matthews KA (1990) Lowering cholesterol concentrations and mortality: a quantitative review of primary prevention trials. Br Med $J$ 301, 309-314.

Muldoon MF, Manuck SB, Mendelsohn AB, Kaplan JR \& Belle SH (2001) Cholesterol reduction and non-illness mortality: meta-analysis of randomised clinical trials. $\mathrm{Br} \mathrm{Med}$ $J$ 322, 11-15.

Ortega RM, Requejo AM, Andres P, et al. (1997) Dietary intake and cognitive function in a group of elderly people. Am J Clin Nutr 66, 803-809.

Osler M, Heitmann BL, Gerdes LU, Jorgensen LM \& Schroll M (2001) Dietary patterns and mortality in Danish men and women: a prospective observational study. $B r J$ Nutr $\mathbf{8 5}$, 219-225.

Paleologos M, Cumming RG \& Lazarus R (1998) Cohort study of vitamin $\mathrm{C}$ intake and cognitive impairment. Am J Epidemiol 148, 45-50.

Pierce JP, Faerber S, Wright FA, et al. (2002) A randomized trial of the effect of a plant-based dietary pattern on additional breast cancer events and survival: the Women's Healthy Eating and Living (WHEL) Study. Control Clin Trials 23, $728-756$.

Plaisted CS, Lin PH, Ard JD, McClure ML \& Svetkey LP (1999) The effects of dietary patterns on quality of life: a substudy of the Dietary Approaches to Stop Hypertension trial. J Am Diet Assoc 99, S84-S89.

Prochaska JO \& Velicer WF (1997) The transtheoretical model of health behavior change. Am J Health Promot 12, 38-48.

Shibata H, Kumagai S, Watanabe S \& Suzuki T (1999) Relationship of serum cholesterols and vitamin $\mathrm{E}$ to depressive status in the elderly. J Epidemiol 9, 261-267.

Smith-Warner SA, Elmer PJ, Tharp TM, et al. (2000) Increasing vegetable and fruit intake: randomized intervention and monitoring in an at-risk population. Cancer Epidemiol Biomarkers Prev 9, 307-317.

Steptoe A, Perkins-Porras L, McKay C, Rink E, Hilton S \& Cappuccio FP (2003a) Behavioural counselling to increase consumption of fruit and vegetables in low income adults: randomised trial. Br Med J 326, 855-858.

Steptoe A, Perkins-Porras L, McKay C, Rink E, Hilton S \& Cappuccio FP (2003b) Psychological factors associated with fruit and vegetable intake and with biomarkers in adults from a low-income neighborhood. Health Psychol 22, $148-155$.

Tiemeier H, Hofman A, Kiliaan AJ, Meijer J \& Breteler MM (2002) Vitamin E and depressive symptoms are not related. The Rotterdam Study. J Affect Disord 72, 79-83.

Toobert DJ, Glasgow RE, Strycker LA, Barrera M Jr, Radcliffe JL, Wander RC \& Bagdade JD (2003) Biologic and quality-of-life outcomes from the Mediterranean Lifestyle Program: a randomized clinical trial. Diabetes Care 26, $2288-2293$.

Toobert DJ, Strycker LA, Glasgow RE, Barrera M \& Bagdade JD (2002) Enhancing support for health behavior change among women at risk for heart disease: the Mediterranean Lifestyle Trial. Health Educ Res 17, 574-585.

Treasure T \& MacRae KD (1998) Minimisation: the platinum standard for trials? Randomisation doesn't guarantee similarity of groups; minimisation does. $\mathrm{Br}$ Med J 317, 362-363.

Vuilleumier J \& Keck E (1989) Fluorometric assay of vitamin C in biological materials using a centrifugal analyser with fluorescence attachment. J Micronutr Anal 5, 25-34.

Wardle J, Parmenter K \& Waller J (2000) Nutrition knowledge and food intake. Appetite 34, 269-275.

Ware JE, Kosinski M \& Keller SD (1994) SF-36 Physical and Mental Health Summary Scales: A User Manual. Boston, MA: The Health Institute, New England Medical Center.

Ware JE \& Sherbourne CD (1992) The MOS 36-item short-form health survey (SF-36). I. Conceptual framework and item selection. Med Care 30, 473-483.

World Health Organization (2002) WHO Mega Country Health Promotion Network. http://www.who.int/hpr/mega 